\title{
Bénard-von Kármán vortex street in an exciton-polariton superfluid
}

\author{
Hiroki Saito, Tomohiko Aioi, and Tsuyoshi Kadokura \\ Department of Engineering Science, University of Electro-Communications, Tokyo 182-8585, Japan
}

(Dated: January 14, 2021)

\begin{abstract}
The dynamics of an exciton-polariton superfluid resonantly injected into a semiconductor microcavity are investigated numerically. The results reveal that a Bénard-von Kármán vortex street is generated in the wake behind an obstacle potential, in addition to the generation of quantized vortex dipoles and dark solitons. The vortex street is shown to be robust against a disorder potential in a sample and it can be observed even in time-integrated measurements.

PACS numbers: 71.36.+c, 47.32.ck, 03.75.Lm, 67.10.Jn
\end{abstract}

\section{INTRODUCTION}

A cylindrical obstacle moving in a classical viscous fluid generates a vortex-antivortex pair in its wake. At large Reynolds numbers, such vortices develop into a periodic pattern known as the Bénard-von Kármán vortex (BvK) street. ${ }^{1,2}$ Such dynamics are also found in superfluids for which vortex circulation is quantized and viscosity is absent. It was numerically demonstrated ${ }^{3}$ that quantized vortex-antivortex pairs, which we call vortex dipoles, are shed in the wake of an obstacle moving in a planar superfluid above a critical velocity. This theoretical prediction has been realized in Bose-Einstein condensates (BECs) of atomic gases, in which quantized vortex dipoles have been created using an moving obstacle potential produced by a laser beam $.4,5$ BvK vortex streets in superfluids (i.e., periodic and alternating creation of quantized vortices and antivortices that form a vortex street with a long lifetime) have also been predicted for atomic $\mathrm{BECs}, \frac{6}{6}$ but they have yet to be demonstrated experimentally.

Exciton-polariton superfluid flow past an obstacle has recently been demonstrated using a semiconductor microcavity ${ }^{7-12}$ In these experiments, polaritons with a controlled in-plane momentum are coherently injected into a sample by a pumping laser, and an obstacle potential is produced by a defect in the microcavity, by a continuous-wave (cw) laser field,, 11 or by etching the sample. 12 A Cherenkov-like pattern and oblique dark solitons are observed for supersonic flow of polariton condensates $\stackrel{8,9}{ }$ Oblique dark solitons formed behind the obstacle decay into quantized vortices. 12 For subsonic flow, quantized vortex dipoles are produced in the wake. ${ }^{9}-11$ These phenomena have been studied theoretically by several researchers $13-16$

A polariton condensate differs from superfluid helium and an atomic gas BEC in that it is a nonequilibrium open system. The polaritons have a short lifetime of $\sim$ $10 \mathrm{ps}$, which is comparable to the time scale of the relevant dynamics. In the experiments described in Refs. 8, 9, polaritons are constantly replenished by pumping with a cw laser and the system reaches a nonequilibrium steady state, in which the pumping balances the loss. Another difference from atomic systems is that the polariton is a coherent superposition of a quantum-well exciton and a cavity photon and the interparticle interaction originates only from the former; in other words, a polariton superfluid is a nontrivial two-component system. Because of these differences, it is by no means obvious that a polariton superfluid shares the same dynamic phenomena as superfluid helium and atomic gas BEC.

In this paper, we investigate the dynamics of an exciton-polariton superfluid passing an obstacle potential and show that a superfluid BvK vortex street $\underline{6}$ emerges in this system, as well as vortex dipoles and dark solitons. We also show that the vortex street is not destroyed by a disorder potential, which is present in a realistic sample. We propose a measurement method to identify a vortex street by time-integrated imaging for the present cw pumped system.

This paper is organized as follows. Section II formulates the problem, Sec. III presents the numerical results, and Sec. IV] gives the conclusions of the study.

\section{FORMULATION OF THE PROBLEM}

We consider a system of quantum-well excitons and cavity photons in the mean-field theory. The mean-field wave functions of excitons $\psi_{\mathrm{X}}$ and photons $\psi_{\mathrm{C}}$ are assumed to obey the two-component nonlinear Schrödinger equation in two dimensions, 13

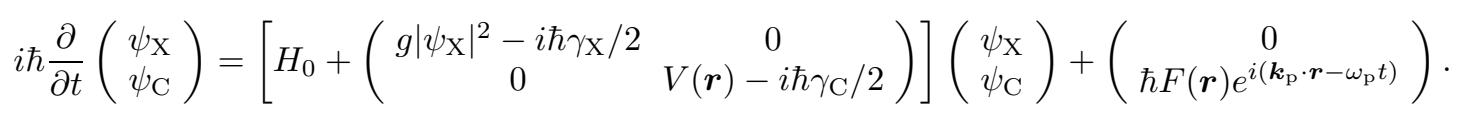

The polariton Hamiltonian in Eq. (11) is given by

$$
H_{0}=\hbar\left(\begin{array}{cc}
\omega_{\mathrm{X}}(-i \nabla) & \Omega_{\mathrm{R}} \\
\Omega_{\mathrm{R}} & \omega_{\mathrm{C}}(-i \nabla)
\end{array}\right)
$$

where the diagonal elements are the dispersion relations of an exciton and a photon, and $\Omega_{\mathrm{R}}$ is the Rabi fre- 
quency of the exciton-photon coupling. In Eq. (1), $g$ is the exciton-exciton interaction coefficient, $\gamma_{\mathrm{X}}$ and $\gamma_{\mathrm{C}}$ are respectively the exciton and photon decay rates, and $V$ is the potential for cavity photons. The last term on the right-hand side of Eq. (11) describes the coherent quasiresonant pumping of photons by an external laser beam with a spatial profile $F$, an in-plane wave vector $\boldsymbol{k}_{\mathrm{p}}$, and a frequency $\omega_{\mathrm{p}}$.

Diagonalizing the noninteracting Hamiltonian in Eq. (2), we obtain the eigenfrequencies of the upper and lower free polaritons as

$$
\omega_{ \pm}=\frac{\omega_{\mathrm{X}}+\omega_{\mathrm{C}} \pm \sqrt{\left(\omega_{\mathrm{X}}-\omega_{\mathrm{C}}\right)^{2}+4 \Omega_{\mathrm{R}}^{2}}}{2} .
$$

The pumping frequency $\omega_{\mathrm{p}}$ is close to $\omega_{-}$to resonantly excite the lower polaritons. We define the detuning as $\delta=\omega_{p}-\omega_{-}\left(\boldsymbol{k}_{\mathrm{p}}\right)$. The exciton is assumed to have the flat dispersion, $\omega_{\mathrm{X}}(\boldsymbol{k})=\omega_{\mathrm{X}}^{0}$, and the dispersion of the cavity photon is approximated to be $\omega_{\mathrm{C}}(\boldsymbol{k})=\omega_{\mathrm{C}}^{0}+\hbar k^{2} /\left(2 m_{\mathrm{C}}\right)$, where $m_{\mathrm{C}}$ is the effective mass of a cavity photon. In the following, we assume $\omega_{\mathrm{X}}^{0}=\omega_{\mathrm{C}}^{0}$.

We numerically solve Eq. (1) using the pseudospectral method, $\frac{17}{2}$ which imposes periodic boundary conditions. The spatial range in the numerical calculation is taken to be sufficiently large that the boundary conditions do not affect the results. The initial state is the vacuum state of excitons and photons, $\psi_{\mathrm{X}}=\psi_{\mathrm{C}}=0$, plus small white noise to break the numerically exact symmetry. The results do not qualitatively depend on the detail of the noise.

\section{NUMERICAL RESULTS}

In the following numerical calculations, we use $m_{\mathrm{C}}=$ $2 \times 10^{-5} m_{\mathrm{e}}$, where $m_{\mathrm{e}}$ is the electron mass, $\hbar \Omega_{\mathrm{R}}=5 \mathrm{meV}$,

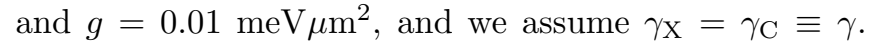
The pumping function $F(\boldsymbol{r})$ is assumed to have the form

$$
F(\boldsymbol{r})= \begin{cases}F_{0} & \left(\alpha x<|y|<y_{\mathrm{p}} \text { and } x>0\right) \\ 0 & (\text { otherwise })\end{cases}
$$

which excites the polaritons in the region of the two triangles shown in Fig. 1.(a). We restrict the pumping area as in Eq. (4), since if the whole space is pumped, the phase will be locked and no vortices will be generated. Similar pumping schemes have been employed in theoretical ${ }^{16}$ and experimental ${ }^{11}$ studies. The parameters in Eq. (44) are taken to be $\hbar F_{0}=38.2 \mathrm{meV}, \alpha=0.4$, and $y_{\mathrm{p}}=50 \mu \mathrm{m}$. The in-plane wave vector of the pumping beam is $\boldsymbol{k}_{\mathrm{p}}=k_{\mathrm{p}} \boldsymbol{e}_{x}$, where $\boldsymbol{e}_{x}$ is the unit vector in the $x$ direction. Polaritons pumped in the triangular areas thus have momentum in the $x$ direction and flow into the region between the triangles, as shown in Fig. 1 (a). An obstacle potential is assumed to be a Gaussian potential as

$$
V(\boldsymbol{r})=V_{0} \exp \left[-\left(x-x_{0}\right)^{2} / d^{2}-y^{2} / d^{2}\right]
$$
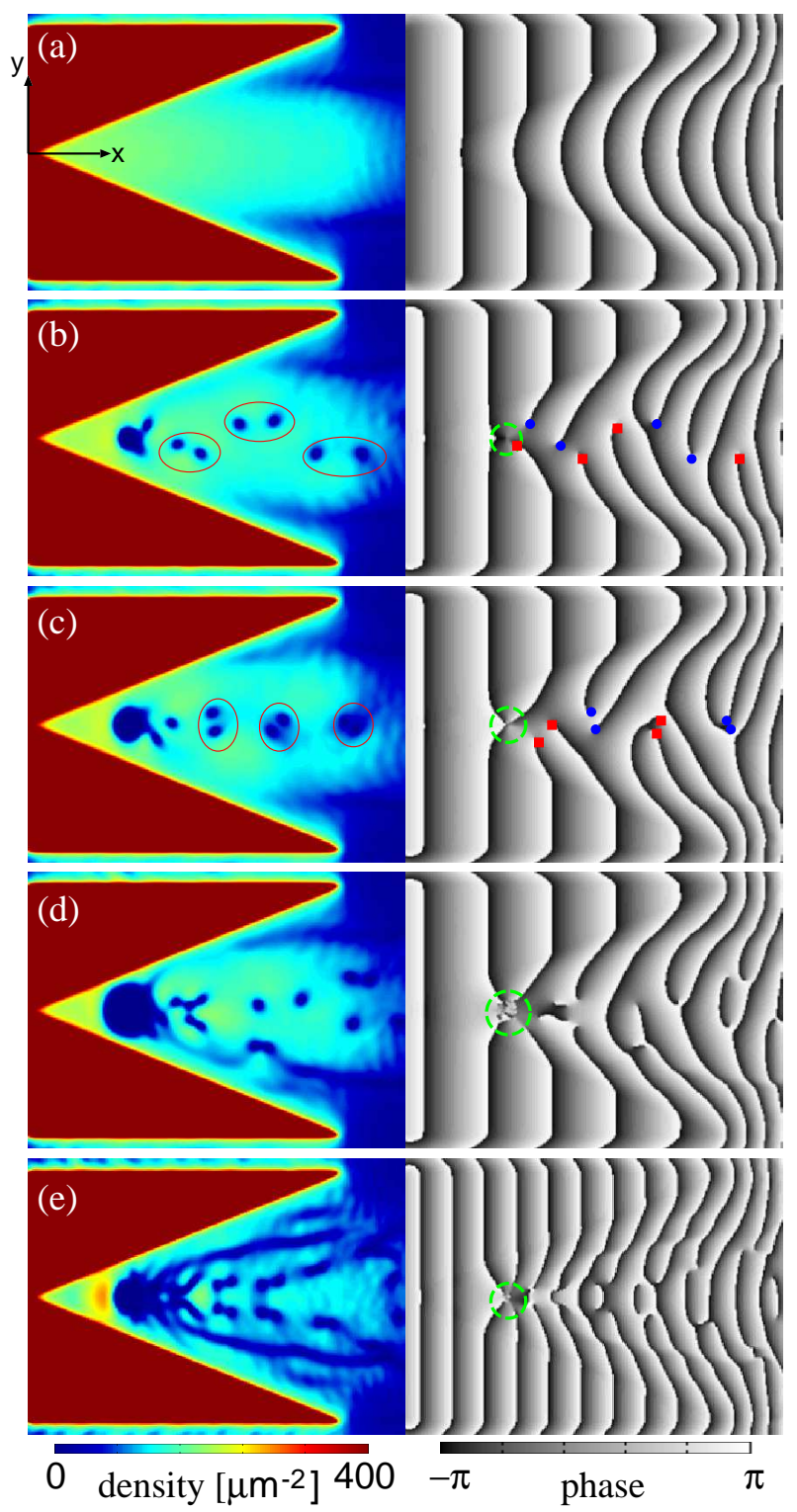

FIG. 1: (Color online) Density $\left|\psi_{\mathrm{C}}\right|^{2}$ and phase $\arg \left(\psi_{\mathrm{C}}\right)$ profiles of the photon wave function at $t=400 \mathrm{ps}$. (a) $k_{\mathrm{p}}=0.25$ $\mu \mathrm{m}^{-1}$ without an obstacle potential, (b) $k_{\mathrm{p}}=0.25 \mu \mathrm{m}^{-1}$ and $d=2 \mu \mathrm{m}$, (c) $k_{\mathrm{p}}=0.25 \mu \mathrm{m}^{-1}$ and $d=3 \mu \mathrm{m},(\mathrm{d}) k_{\mathrm{p}}=0.25$ $\mu \mathrm{m}^{-1}$ and $d=5 \mu \mathrm{m}$, and (e) $k_{\mathrm{p}}=0.5 \mu \mathrm{m}^{-1}$ and $d=3 \mu \mathrm{m}$. The solid circles in (b) and (c) respectively indicate vortex dipoles and co-rotating twin vortices, which are released from the obstacle potential. The dashed circles indicate the locations of the obstacle potential and the filled blue circles and red squares indicate clockwise and counterclockwise vortices, respectively. The detuning is $\hbar \delta=0.7 \mathrm{meV}$ and the lifetime is $\gamma^{-1}=30 \mathrm{ps}$. The field of view of each panel is $150 \times 110 \mu \mathrm{m}$ and the origin is located at the center of the left edge of the panel. See Supplemental Material for movies of the dynamics in (b)-(e). 
with $V_{0}=38.2 \mathrm{meV}$ and $x_{0}=40 \mu \mathrm{m}$, which is located between the triangles. Thus, polaritons flowing between the triangular areas hit the obstacle potential and generate a wake in the $x$ direction. The healing length and the Bogoliubov sound speed near the obstacle potential are respectively estimated to be $\xi \simeq \hbar /\left(m_{\mathrm{LP}} g_{\mathrm{LP}} n_{\mathrm{LP}}\right)^{1 / 2} \simeq 1.5$ $\mu \mathrm{m}$ and $v_{\mathrm{s}} \simeq\left(g_{\mathrm{LP}} n_{\mathrm{LP}} / m_{\mathrm{LP}}\right)^{1 / 2} \simeq 1.8 \times 10^{6} \mathrm{~m} / \mathrm{s}$, where $m_{\mathrm{LP}}, g_{\mathrm{LP}}$, and $n_{\mathrm{LP}}$ are respectively the mass, the effective coupling constant, 13 and the density of the lower polariton.

Figure 1 shows profiles of the photon wave function $\psi_{\mathrm{C}}$ at $t=400 \mathrm{ps}$. In Fig. 11 (b), quantized vortex dipoles are generated behind the obstacle potential. After the vortex dipoles are released from the potential, they alternately align in the wake, as shown in Fig. 1 (b). Such alternate alignment of vortex dipoles in superfluids is also observed in Refs. 6, 18. Figure 1 (c) shows the situation for a larger obstacle potential. In this case, two vortices with the same circulation form a pair and clockwise and counterclockwise pairs are shed alternately. This vortex shedding dynamics is very similar to that in Ref. $\underline{6}$, which is a superfluid analogue of the BvK vortex street. This periodic vortex configuration has a very long lifetime ${ }^{6}$ when there is no dissipation or losses. Thus, a small obstacle generates vortex dipoles and a large obstacle generates a BvK vortex street, which is consistent with the case of an atomic BEC (Fig. 3 of Ref. 6). For a larger obstacle potential, vortex shedding becomes irregular as shown in Fig. 1 (d). The flow velocity in Figs. 1 (a)-1 (d) is $\hbar k_{\mathrm{p}} / m_{\mathrm{LP}} \simeq 0.4 v_{\mathrm{s}}$, which is comparable to the critical velocity for vortex nucleation obtained from the Gross-Pitaevskii equation. ${ }^{3}$ Figure 1 (e) shows the case for faster flow with flow velocity of $\simeq 0.7 v_{\mathrm{s}}$. Dark solitons and vortex dipoles are generated in the wake, as experimentally observed 12 The vortex patterns shown in Fig. 1 can be observed for a shorter polariton lifetime (e.g., 15 ps) if the polariton density near the obstacle is kept large by increasing the pump intensity or narrowing the space between the triangular pumped regions (data not shown). For all the cases in Fig. 1, the exciton wave function $\psi_{\mathrm{X}}$ has a similar profile to that of the photon wave function $\psi_{\mathrm{C}}$.

In microcavity samples used in experiments, a disorder potential is inevitable; it is of the order of $0.1 \mathrm{meV}$, even for a good sample. 19 Figure 2 shows the effect of the disorder potential on vortex street formation, where the potential shown in Fig. 2(b) is added to $V(\boldsymbol{r})$ in Eq. (1) while the other parameters remain the same as those in Fig. 1 (c). Figure 2 (a) shows that the BvK vortex street is robust against a disorder potential in a realistic sample.

In the present situation, it is impossible to perform time-resolved measurements, as were performed in Refs. 8, 10, 12 For time-resolved measurements, polaritons must be pumped by a pulsed laser beam, which is split and used as a reference in the interferometer with a delay time, enabling time-resolved images to be obtained by performing repeated measurements. On the other hand, polaritons are pumped by a cw laser in the present system (a)

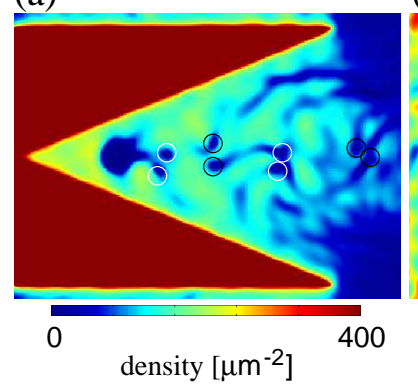

(b)

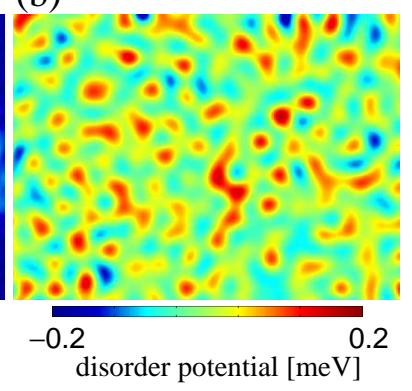

FIG. 2: (Color online) (a) Density profile $\left|\psi_{\mathrm{C}}\right|^{2}$ in the presence of the disorder potential shown in (b). The disorder potential is generated by setting random numbers on each site and cutting off short-wavelength Fourier components. The parameters are the same as those in Fig. 1(c). The black and white circles indicate clockwise and counterclockwise vortices, respectively. See the Supplemental Material for a movie of the dynamics.
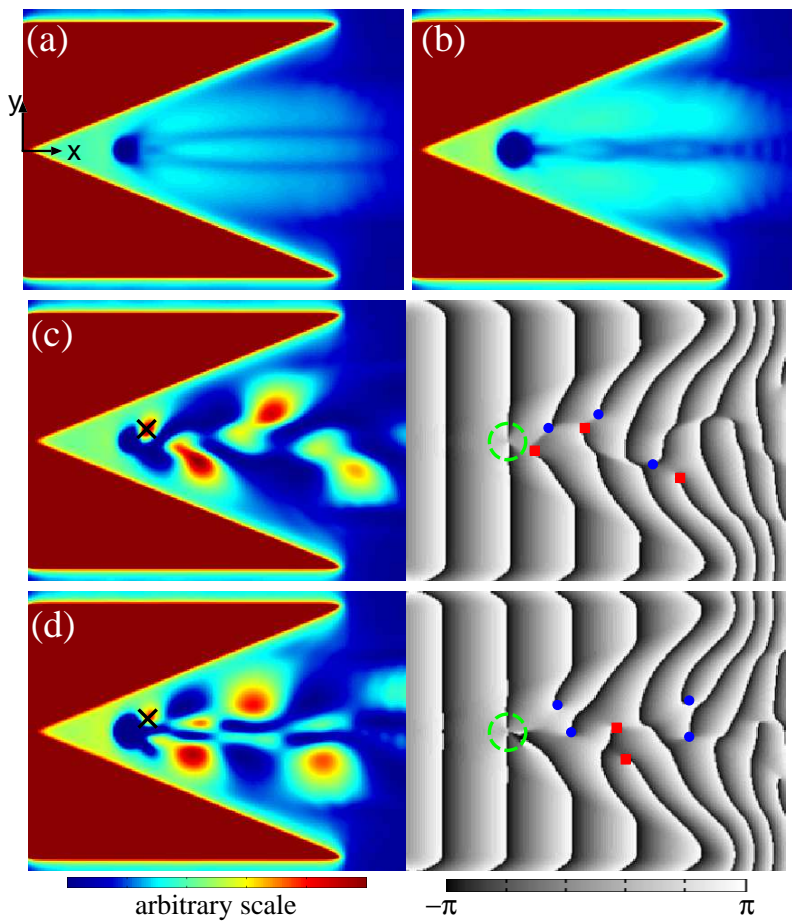

FIG. 3: (Color online) (a) Time-integrated density of the photon wave function $I(\boldsymbol{r})=\int\left|\psi_{\mathrm{C}}(\boldsymbol{r}, t)\right|^{2} d t$ for the dynamics in Fig. 1(b). (b) $I$ for the dynamics in Fig. 10(c). (c) Density $|G|^{2}$ (left) and phase $\arg (G)$ (right) of the first-order coherence $G(\boldsymbol{r})=\int \psi_{\mathrm{C}}^{*}\left(\boldsymbol{r}_{\mathrm{ref}}, t\right) \psi_{\mathrm{C}}(\boldsymbol{r}, t) d t$ for the dynamics in Fig. 1 (b). (d) $|G|^{2}$ (left) and $\arg (G)$ (right) for the dynamics in Fig. 1(c). The time integrations in $I$ and $G$ are taken between $t=400$ and $1400 \mathrm{ps}$ (the patterns are independent of the upper integration limit if the time integration is sufficiently long). The position of the reference is $\left(x_{\text {ref }}, y_{\text {ref }}\right)=(46,4)$, which is marked by the crosses. The field of view of each panel is $150 \times 110 \mu \mathrm{m}$ and the origin is located at the center of the left edge of the panel. 
and hence only time-integrated images can be obtained. Figures 3 (a) and 3 (b) show time-integrated density profiles $\int\left|\psi_{\mathrm{C}}(\boldsymbol{r}, t)\right|^{2} d t$ of the dynamics in Figs. 11 (b) and 1 (c), respectively. Although the traces of vortex flow are visible, individual vortices are smeared out and cannot be discerned. $\frac{9}{}$ To overcome this problem, we examine the time-integrated spatial coherence given by

$$
G(\boldsymbol{r})=\int \psi_{\mathrm{C}}^{*}\left(\boldsymbol{r}_{\mathrm{ref}}, t\right) \psi_{\mathrm{C}}(\boldsymbol{r}, t) d t,
$$

where $\boldsymbol{r}_{\text {ref }}$ is the position of the reference light source. The reference light from a small area at $\boldsymbol{r}_{\text {ref }}$ is enlarged and interference with the whole image is measured using an interferometer with a variable arm length $\underline{\underline{9}}$

Figures 3 (c) and 3 (d) show the density and phase profiles of $G$ for the dynamics in Figs. 11 (b) and 11 (c), respectively. The reference $\boldsymbol{r}_{\text {ref }}$ is positioned obliquely behind the obstacle (crosses in Fig. 3), where the density oscillates with time due to periodic vortex shedding. Therefore, time integration of $G$ is performed stroboscopically at the same frequency as the change in the vortex pattern, preserving the information of vortices, as shown in Figs. 3 (c) and 3 (d). Figure 3 (c), which corresponds to the vortex dipole generation in Fig. 1 (b), exhibits vortex dipoles in the phase profiles (left panel of Fig. 3 (c)). The phase profile in Fig. 3 (d) also reflects the BvK vortex street in Fig. 1 (c). Thus, the time-integrated coherence $G$ contains information on the vortex patterns and it enables us to identify vortex street formation ${ }^{20}$

\section{CONCLUSIONS}

We have investigated the dynamics of excitonpolariton superfluid flow passing an obstacle potential in a semiconductor microcavity. Numerically solving the two-component nonlinear Schrod̈inger equation in Eq. (11) reveals a superfluid BvK vortex street (Fig.1(c)), as well as alternately aligned vortex dipoles (Fig. 1 (b)) and dark solitons (Fig. 1 (d)). The formation of the vortex street is robust against a disorder potential, which is naturally present in samples (Fig. 2). These periodic vortex patterns are time-dependent steady states that are attained by cw pumping of polaritons and time-resolved imaging cannot be performed. We showed that the spatial coherence in Eq. (6) with an appropriate reference point $\boldsymbol{r}_{\text {ref }}$ reflects the vortex patterns even though it is time integrated (Fig. 3). We have thus demonstrated that a superfluid BvK vortex street can be generated and detected in an exciton-polariton condensate using current experimental techniques.

\section{Acknowledgments}

This work was supported by Grants-in-Aid for Scientific Research (No. 22340116 and No. 23540464) from the Ministry of Education, Culture, Sports, Science and Technology of Japan.
1 H. Bénard, C. R. Acad. Sci. Paris 147, 839 (1908); 147, 970 (1908).

2 T. von Kármán, Nachr. Ges. Wiss. Göttingen, Math. Phys. Kl. 509 (1911); 547 (1912).

3 T. Frisch, Y. Pomeau, and S. Rica, Phys. Rev. Lett. 69, 1644 (1992).

4 S. Inouye, S. Gupta, T. Rosenband, A. P. Chikkatur, A. Görlitz, T. L. Gustavson, A. E. Leanhardt, D. E. Pritchard, and W. Ketterle, Phys. Rev. Lett. 87, 080402 (2001).

5 T. W. Neely, E. C. Samson, A. S. Bradley, M. J. Davis, and B. P. Anderson, Phys. Rev. Lett. 104, 160401 (2010).

${ }^{6}$ K. Sasaki, N. Suzuki, and H. Saito, Phys. Rev. Lett. 104, 150404 (2010).

7 A. Amo, D. Sanvitto, F. P. Laussy, D. Ballarini, E. del Valle, M. D. Martin, A. Lemaître, J. Bloch, D. N. Krizhanovskii, M. S. Skolnick, C. Tejedor, and L. Viña, Nature 457, 291 (2009).

8 A. Amo, J. Lefrére, S. Pigeon, C. Adrados, C. Ciuti, I. Carusotto, R. Houdré, E. Giacobino, and A. Bramati, Nature Phys. 5, 805 (2009).

9 A. Amo, S. Pigeon, D. Sanvitto, V. G. Sala, R. Hivet, I. Carusotto, F. Pisanello, G. Leménager, R. Houdré, E. Giacobino, C. Ciuti, and A. Bramati, Science 332, 1167 (2011).

10 G. Nardin, G. Grosso, Y. Léger, B. Piętka, F. MorierGenoud, and B. Deveaud-Plédran, Nature Phys. 7, 635
(2011).

11 D. Sanvitto, S. Pigeon, A. Amo, D. Ballarini, M. De Giorgi, I. Carusotto, R. Hivet, F. Pisanello, V. G. Sala, P. S. S. Guimaraes, R. Houdré, E. Giacobino, C. Ciuti, A. Bramati, and G. Gigli, Nature Photonics 5, 610 (2011).

12 G. Grosso, G. Nardin, F. Morier-Genoud, Y. Léger, and B. Deveaud-Plédran, Phys. Rev. Lett. 107, 245301 (2011).

13 I. Carusotto and C. Ciuti, Phys. Rev. Lett. 93, 166401 (2004).

14 M. Wouters and I. Carusotto, Phys. Rev. Lett. 105, 020602 (2010).

15 E. Cancellieri, F. M. Marchetti, M. H. Szymańska, and C. Tejedor, Phys. Rev. B 82, 224512 (2010).

16 S. Pigeon, I. Carusotto, and C. Ciuti, Phys. Rev. B 83, 144513 (2011).

17 W. H. Press, S. A. Teukolsky, W. T. Vetterling, B. P. Flannery, Numerical Recipes, 3rd ed, Sec. 20.7 (Cambridge Univ. Press, Cambridge, 2007).

18 C. Nore, M. E. Brachet, and S. Fauve, Physica D 65, 154 (1993).

19 G. Roumpos, M. D. Fraser, A. Löffler, S. Höfling, A. Forchel, and Y. Yamamoto, Nature Phys. 7, 129 (2010).

20 Recently, a similar scheme to detect a vortex lattice is proposed in M. O. Borgh, G. Franchetti, J. Keeling, and N. Berloff, arXiv:1204.4095 